\title{
The Effectiveness and Usability of Online, Group-Based Interventions for People With Severe Obesity: Protocol for a Systematic Review
}

Madison Milne-Ives ${ }^{1}$, BAS, MSc; Dawn Swancutt ${ }^{2}$, BSc, MPH, PhD; Lorna Burns ${ }^{3}$, BA, MSc; Jonathan Pinkney ${ }^{2}$, BSc, MBBS, MRCP, MD, FRCP; Mark Tarrant ${ }^{4}$, BA, PhD; Raff Calitri ${ }^{4}$, BSc, MSc, PhD; Arunangsu Chatterjee ${ }^{1}$, BEng, MSc, PhD; Edward Meinert ${ }^{1,5}$, MA, MSc, MBA, MPA, PhD, CEng, FBCS, EUR ING

\footnotetext{
${ }^{1}$ Centre for Health Technology, University of Plymouth, Plymouth, United Kingdom

${ }^{2}$ Peninsula Medical School, Faculty of Health, University of Plymouth, Plymouth, United Kingdom

${ }^{3}$ Peninsula Dental School, Faculty of Health, University of Plymouth, Plymouth, United Kingdom

${ }^{4}$ Exeter Medical School, College of Medicine and Health, University of Exeter, Exeter, United Kingdom

${ }^{5}$ Department for Primary Care and Public Health, Imperial College London, London, United Kingdom
}

\section{Corresponding Author:}

Edward Meinert, MA, MSc, MBA, MPA, PhD, CEng, FBCS, EUR ING

Centre for Health Technology

University of Plymouth

6 Kirkby Place

Plymouth, PL4 6DT

United Kingdom

Phone: 441752600600

Email: edward.meinert@plymouth.ac.uk

\begin{abstract}
Background: Globally, obesity is a growing crisis. Despite obesity being preventable, over a quarter of the UK adult population is currently considered clinically obese (typically body mass index $\geq 35 \mathrm{~kg} / \mathrm{m}^{2}$ ). Access to treatment for people with severe obesity is limited by long wait times and local availability. Online and group-based interventions provide means of increasing the accessibility of obesity prevention and treatment services. However, there has been no prior review of the effectiveness of group-based interventions delivered online for people with severe obesity.
\end{abstract}

Objective: The purpose of this systematic review protocol is to provide an evaluation of the effectiveness and usability of different types of online, group-based interventions for people with severe obesity.

Methods: The Preferred Reporting Items for Systematic Reviews and Meta-Analyses Protocols (PRISMA-P) and the Population, Intervention, Comparator, Outcome, and Study (PICOS) frameworks were used to structure this review. The review will systematically search 7 databases: MEDLINE, Embase, the Cumulative Index of Nursing and Allied Health Literature, APA PsycNet, Web of Science, CENTRAL, and the ProQuest Dissertations and Theses databases. Two authors (MM-I and LB) will independently screen the titles and abstracts of identified articles, select studies for inclusion based on the eligibility criteria, and extract data into a standardized form. Any disagreements will be discussed and resolved by a third reviewer (EM) if necessary. Risk of bias will be assessed using the Cochrane Collaboration Risk of Bias 2 tool and a descriptive analysis will be used to evaluate effectiveness and usability.

Results: The systematic review has not yet been started. It is expected to be completed and submitted for publication by December 2021.

Conclusions: This systematic review will summarize the effectiveness and usability of online, group-based interventions for people with obesity. It will identify the types of online delivery that have the strongest support to help inform the development of more useful and engaging interventions for people with severe obesity.

Trial Registration: National Institute for Health Research, PROSPERO CRD42021227101; https://www.crd.york.ac.uk/prospero/display_record.php?ID=CRD42021227101

International Registered Report Identifier (IRRID): PRR1-10.2196/26619 
(JMIR Res Protoc 2021;10(6):e26619) doi: 10.2196/26619

\section{KEYWORDS}

internet-based interventions; telemedicine; group-based interventions; obesity; severe obesity; obesity management; weight loss; weight reduction programs; diet therapy; exercise; systematic review; weight management

\section{Introduction}

\section{Background}

Obesity is a serious and growing crisis; over a quarter of UK adults are considered obese ( $26 \%$ of men and $29 \%$ of women) [1]. Adults with a BMI greater than or equal to $35 \mathrm{~kg} / \mathrm{m}^{2}$ are considered to have a very high health risk [2]. This is a significant concern, as obesity has been linked to several physical and mental health conditions, such as type 2 diabetes, heart disease, cancer, stroke, and depression [3,4]. The COVID-19 pandemic has only made obesity a more urgent health issue to tackle. Evidence to this point has identified a notably increased risk of severe COVID-19 symptoms, need for hospitalization and intensive care, and increased mortality for patients who are overweight and obese [5]. This has highlighted the need for sustained and effective interventions to improve physical activity and dietary behaviors to prevent a worsening impact on health outcomes [5].

The UK has a 4-tiered pathway for obesity services: tier 1 refers to universal obesity prevention services, tier 2 covers community-based lifestyle weight management services, tier 3 is specialist obesity services (provided by a multidisciplinary team), and tier 4 is surgery [6,7]. The higher tiers (3 and 4) are specifically targeted toward adults with high BMIs $\left(\geq 40 \mathrm{~kg} / \mathrm{m}^{2}\right.$, or $35 \mathrm{~kg} / \mathrm{m}^{2}$ with a comorbidity) [4].

In practice, access to these services is not universal. An All-Party Parliamentary Group found that more than one-third of people with obesity had not accessed any of these services, and almost $40 \%$ of those who did found the process moderately or incredibly difficult [8]. Additionally, of the $91 \%$ of Clinical CoMM-Issioning Groups (that tier 3 services fall under) that responded to a freedom of information request, less than $60 \%$ coMM-Issioned tier 3 services [8]. Likewise, in a survey conducted by Public Health England, people reported long waiting lists and a lack of local availability for tier 3 services [9]. A longitudinal cohort study found that almost three-quarters of people with severe obesity (BMI $\geq 35 \mathrm{~kg} / \mathrm{m}^{2}$ ) did not access any treatment over the course of 7 years; this figure was lower, but still high (almost 60\%), for people with morbid obesity (BMI $\geq 40 \mathrm{~kg} / \mathrm{m}^{2}$ ) [10]. These statistics highlight a significant problem with access to and availability of obesity prevention services in the UK.

\section{Rationale}

There are several strategies that can help address the need for greater accessibility of interventions for people with severe obesity. Group-based interventions may improve accessibility by potentially reducing the resources needed to provide interventions by supporting more patients with fewer staff hours required. This also has the potential to reduce waiting times to access interventions [4]. Group-based interventions are a common tool to promote health behavior change [11] and previous systematic reviews have found that group-based interventions are generally effective at promoting physical activity and weight loss $[12,13]$.

Another strategy that is increasingly used to improve accessibility to a variety of health interventions is the use of digital and online platforms of delivery. Online delivery strategies have become increasingly common as COVID-19 restrictions have forced services to adapt the way they provide support [14]. A wide variety of platforms have been used to deliver services, with mixed feedback from participants [14]. A previous systematic review found evidence suggesting that web-based interventions are more effective at promoting and maintaining weight loss than minimal or no interventions, but that evidence of their effectiveness compared with in-person interventions is mixed [15]. Another systematic review found that online interventions were more effective at promoting weight loss in the short term, but not the long term, compared with offline interventions [16]. A systematic review of mobile-based interventions for people who are overweight or obese also found evidence of their effectiveness in primary and secondary health care settings [17], but evidence for their effectiveness in general is mixed [18].

This suggests that online platforms have potential to support effective weight loss interventions, but that further research is needed to determine best practice.

Combining these 2 strategies (ie, group based and web based) could further improve the accessibility of interventions, particularly given the COVID-19 restrictions on in-person interactions. However, no previous systematic reviews were identified that examined online, group-based interventions for people with obesity. One systematic review did review online social network weight management interventions, but only included 5 studies and was focused specifically on social networks without including other types of online interventions [19]. Several searches of keywords ("online OR digital," "group-based interventions OR group interventions OR group behaviour change," and "weight loss OR obesity") on PROSPERO failed to identify any systematic reviews that are currently exploring the effectiveness of different types of online, group-based interventions for people with obesity.

Therefore, there is a need for a systematic review of studies that evaluate online, group-based interventions for people with severe obesity to determine their effectiveness, usability, and the conditions which make them most effective and engaging for participants. An overview of the different types of online, group-based weight loss interventions will make it easier to identify best practices and contribute to the development of new online, group-based interventions that can best promote and maintain weight loss. 
To focus the evaluation, this systematic review will examine 2 main research questions. First, what means of delivering online, group-based behavior change interventions for adults with severe obesity is the most effective at establishing and maintaining positive health behavior changes and weight loss? Second, what are the perceptions of the acceptability, usability, and overall user experience for different online, group-based behavior change interventions for adults with severe obesity?

\section{Methods}

\section{Overview}

The Population, Intervention, Comparator, Outcome, and Study (PICOS) template and the Preferred Reporting Items for
Systematic Reviews and Meta-Analyses Protocols (PRISMA-P) [20] will be used to structure the review and to identify appropriate Medical Subject Headings (MeSH) for the literature search (Multimedia Appendix 1). This systematic review will be composed of a literature search, article selection, data extraction, quality appraisal, data analysis, and data synthesis. This review was prospectively registered on PROSPERO (reference number: CRD42021227101).

\section{Eligibility Criteria}

The PICOS-type framework (Table 1) is based on the research question stated above.

Table 1. PICOS framework.

\begin{tabular}{ll}
\hline Framework component & Description \\
\hline Population & Adults ( $\geq 18$ years) with severe obesity (defined for this review as BMI $\geq 35 \mathrm{~kg} / \mathrm{m}^{2}$ ) \\
Intervention & Online, group-based interventions aiming to change health behavior relating to obesity (physical activity and dietary behavior) \\
Comparator & $\begin{array}{l}\text { Other types of group-based interventions, including comparisons with face-to-face, phone, and other online platforms than } \\
\text { the main intervention }\end{array}$ \\
Outcomes & $\begin{array}{l}\text { The primary objective is to identify the types of online platforms used for group-based interventions for people with obesity } \\
\text { and their effectiveness. Therefore, the primary outcomes will be the effectiveness of the interventions at supporting behavior } \\
\text { changes (physical activity and dietary behavior) and weight loss. Secondary outcomes will include levels of engagement } \\
\text { with the intervention, and patient-reported experience (including measures of acceptability, usability, or satisfaction). Other } \\
\text { secondary outcomes - including details about the intervention design, aim, and format-will also be examined. }\end{array}$
\end{tabular}

Study types

Studies that evaluate at least one online, group-based intervention for people with severe obesity will be eligible (including randomized controlled trials, quantitative, qualitative, cohort, and case studies). Reviews, protocols, and papers that describe interventions without evaluating them will be excluded.

\section{Search Strategy}

Seven databases will be searched to find articles for this review: MEDLINE, Embase, CINAHL, APA PsycNet, Web of Science, the CENTRAL, and the ProQuest Dissertations and Theses databases. Key terms relating to online, group-based interventions for people with severe obesity were extracted from an initial review of the literature and used to develop the search terms and search strategy. Search terms will include MeSH terms and title / abstract keywords relating to online interventions, group-based interventions, and severe obesity. The search terms that will be used in this review are grouped into those 3 themes (Table 2) and the search string will be created using the following structure: online (MeSH OR Keywords) AND group-based (MeSH OR Keywords) AND severe obesity (MeSH OR Keywords). No date limit will be set. See Multimedia Appendix 2 for a sample search string.

Table 2. Search terms.

\begin{tabular}{|c|c|c|}
\hline Component & $\mathrm{MeSH}$ & Keywords (in title or abstract) \\
\hline Online & $\begin{array}{l}\text { Internet-based intervention/ OR internet/ } \\
\text { OR telemedicine/ OR videoconferencing/ }\end{array}$ & Internet OR web OR online OR remote OR digital OR video* OR virtual OR technolog* \\
\hline Group-based & $\begin{array}{l}\text { Psychotherapy, Group/ OR Peer Group/ } \\
\text { OR Group Processes/ }\end{array}$ & $\begin{array}{l}\text { (group* adj3 (based OR treatment* OR therap* OR virtual OR session* OR peer* OR } \\
\text { support*)) OR "group intervention" }\end{array}$ \\
\hline Severe obesity & exp obesity/ OR obesity management/ & $\begin{array}{l}\text { obesity OR obese OR specialist weight management OR Tier } 3 \text { weight management OR } \\
\text { (BMI adj1 ("35" OR " } 40 \text { " OR “45")) }\end{array}$ \\
\hline
\end{tabular}

\section{Inclusion Criteria}

The review will include studies that evaluate online, group-based interventions for people with severe obesity (defined for this review as BMI $\geq 35 \mathrm{~kg} / \mathrm{m}^{2}$ ). Online, group-based interventions will be defined as an intervention delivered primarily online to a set of 3 or more people. Interventions that are primarily group based will be included, even if they have a small individual component. Interventions will need to target weight management for people with obesity but can focus on behavioral (eg, diet, physical activity) or physical (eg, weight loss or maintenance of weight loss) components. Interventions with comparisons to control groups with no intervention, waiting list or irrelevant interventions, minimal interventions, usual care, in-person interventions, or other online interventions will all be included. 


\section{Exclusion Criteria}

Studies of children and adolescents (participants aged < 18) will be excluded. Studies that address diet or physical activity behavior changes with a primary purpose other than managing obesity or weight loss (eg, rehabilitation after surgery, chronic obstructive pulmonary disease, diabetes) will also be excluded. Interventions that are primarily one-on-one but have a small group-based component will be excluded.

\section{Screening and Article Selection}

The references identified from the database searches will be exported into a citation management software (EndNote X9; Clarivate Analytics) for storage and duplicate removal. There will be 2 stages of screening: (1) 2 independent reviewers (MM-I

Textbox 1. Article information and data extraction. and LB) will screen the titles and abstracts and (2) 2 independent reviewers will screen the full text of the studies to determine final eligibility for inclusion. All disagreements between the reviewers will be discussed and a third reviewer (EM) will be consulted if consensus cannot be reached. A PRISMA flow diagram will be used to record the details of the screening and selection process to ensure study reproducibility.

\section{Data Extraction}

Two independent reviewers (MM-I and another reviewer) will examine the full text of all of the included articles to extract outcomes into a predetermined form (Textbox 1). Any disagreements between the reviewers will be discussed and resolved by a third reviewer (EM) if consensus cannot be reached.

\footnotetext{
General study information

- Year of publication

- Country of study

- Sample demographics (including age, gender, target population)

- Initial sample size

- Analyzed sample size

Intervention

- Online platform

- Aim of intervention (eg, increase physical activity, improve dietary behavior)

- Group size

- Number and length of intervention sessions

- Intervention duration and follow-up periods

- Theory the intervention is based on (if any)

- Behavior change techniques [21] used in the intervention (if any)

Evaluation

- Outcomes measured

- Effect of intervention on behavior change outcomes

- Effect of intervention on health outcomes (eg, weight, BMI)

- Participant engagement (eg, drop-out rates, number of sessions attended)

- Acceptability

- Usability

- Participant satisfaction/feedback

- Other key performance indicators reported
}

\section{Quality Appraisal and Risk of Bias Assessment}

Two reviewers (MM-I and another reviewer) will independently assess the risk of bias of all of the included studies. Any disagreements will be discussed or resolved by a third reviewer (EM). The risk of bias of the randomized controlled trials will be assessed using the Cochrane Collaboration Risk of Bias 2 tool to assign high or low risk of bias, or some concerns [22,23]. Nonrandomized studies will be assessed using the ROBINS-I tool [24]. Figures will be created to summarize the risk of bias in each study and the extent of each bias across all studies.

\section{Data Analysis and Synthesis}

Because of the expected variety of study aims, measures, and reported outcomes, it is not likely that a meta-analysis will be feasible. However, the feasibility of a meta-analysis will be considered when studies have been assessed. A descriptive analysis will be conducted to summarize the extracted data. 
General study information will be summarized in a table. Outcomes relating to the intervention will be synthesized quantitatively (eg, by providing the percentage of studies that used a particular platform, theory, or behavior change technique and had a particular aim and by providing the mean, median, and range for outcomes such as group size, number of sessions, and session duration).

The primary outcomes-the effect of the intervention on behavior change and health outcomes — will be quantified by providing the percentage of studies that found significant evidence of effectiveness. Substantial evidence of effectiveness will be defined as a significantly better performance of the intervention than the comparator. Behavior change and physical health outcomes will be considered separately.

Analysis of other outcomes, such as acceptability, usability, and patient feedback will be determined upon review of the studies, as they could be analyzed qualitatively or quantitatively depending on what is reported. Any qualitative data reported by the study will be assessed using a thematic analysis to identify similarities and differences in participant responses to the interventions. The risk of bias in the studies will be considered in the synthesis.

\section{Results}

The full systematic review is expected to be completed and submitted for publication by December 2021.

\section{Discussion}

A systematic review of the literature on online, group-based behavior change interventions for adults with severe obesity will contribute to the establishment of guidelines for best practice. With the strained capacity to provide specialist weight management services (also known as tier 3 services), and the additional constraints of the COVID-19 pandemic on group-based and face-to-face services, a better understanding of how group-based interventions can be effectively delivered remotely will help inform and improve the development of online interventions for adults with severe obesity in the UK. Based on the data, this section will explore what conclusions can be drawn, the limitations of the systematic review, and key topics for future research.

\section{Acknowledgments}

This work is independent research supported by the National Institute for Health Research Applied Research Collaboration South West Peninsula. The views expressed in this publication are those of the author(s) and not necessarily those of the National Institute for Health Research or the Department of Health and Social Care.

\section{Authors' Contributions}

The review topic was conceived by all the authors collectively. MM-I wrote the protocol with revisions from DS, LB, JP, MT, $\mathrm{RC}, \mathrm{AC}$, and EM.

\section{Conflicts of Interest}

None declared.

\section{Multimedia Appendix 1}

PRISMA-P checklist. [DOC File, 82 KB-Multimedia Appendix 1]

\section{Multimedia Appendix 2}

Sample search.

\section{[DOCX File, 15 KB-Multimedia Appendix 2]}

\section{References}

1. NHS. Statistics on Obesity, Physical Activity and Diet, England. 2020. URL: https://digital.nhs.uk/data-and-information/ publications/statistical/statistics-on-obesity-physical-activity-and-diet/england-2020 [accessed 2020-11-29]

2. NHS. Part 3: Adult Overweight and Obesity. 2020. URL: https://digital.nhs.uk/data-and-information/publications/statistical/ statistics-on-obesity-physical-activity-and-diet/england-2020/part-3-adult-obesity-copy [accessed 2020-11-29]

3. NHS. Obesity. URL: https://www.nhs.uk/conditions/obesity/ [accessed 2020-11-29]

4. Swancutt D, Tarrant M, Pinkney J. How Group-Based Interventions Can Improve Services for People with Severe Obesity. Curr Obes Rep 2019 Sep;8(3):333-339 [FREE Full text] [doi: 10.1007/s13679-019-00348-y] [Medline: 31062202]

5. Blackshaw J, Feeley A, Mabbs L, Niblett P, Atherton E, Elsom R, et al. Excess Weight and COVID-19: Insights From New Evidence. London, UK: Public Health England; 2020 Jul. URL: https://assets.publishing.service.gov.uk/government/ uploads/system/uploads/attachment data/file/907966/PHE insight Excess weight and COVID-19 FINAL.pdf [accessed 2020-11-29] 
6. National Institute for Health and Care Excellence. Weight management: lifestyle services for overweight or obese adults (Report No.: Public health guideline [PH53]). London, UK: National Institute for Health and Care Excellence; 2014 May. URL: https://www.nice.org.uk/guidance/ph53 [accessed 2020-11-29]

7. Capehorn MS, Haslam DW, Welbourn R. Obesity Treatment in the UK Health System. Curr Obes Rep 2016 Sep 28;5(3):320-326. [doi: 10.1007/s13679-016-0221-z] [Medline: 27352180]

8. Selous A, Finlay I, Jenkin A, Smith E, Warner N. The current landscape of obesity services: a report from the All-Party Parliamentary Group on Obesity. 2018. URL: http://www.bomss.org.uk/wp-content/uploads/2018/05/APPG-Obesity-2018. pdf [accessed 2021-03-15]

9. Public Health England. National mapping of weight management services: Provision of tier 2 and tier 3 services in England. London, UK: Public Health England; 2015 Dec. URL: https://assets.publishing.service.gov.uk/government/uploads/system/ uploads/attachment data/file/484115/Final Weight Management Mapping Report.pdf [accessed 2021-03-15]

10. Booth HP, Prevost AT, Gulliford MC. Access to weight reduction interventions for overweight and obese patients in UK primary care: population-based cohort study. BMJ Open 2015 Jan 13;5(1):e006642 [FREE Full text] [doi: 10.1136/bmjopen-2014-006642] [Medline: 25586371]

11. Borek AJ, Abraham C, Greaves CJ, Gillison F, Tarrant M, Morgan-Trimmer S, et al. Identifying change processes in group-based health behaviour-change interventions: development of the mechanisms of action in group-based interventions (MAGI) framework. Health Psychol Rev 2019 Jun 13:1-21. [doi: 10.1080/17437199.2019.1625282] [Medline: 31190619]

12. Borek AJ, Abraham C, Greaves CJ, Tarrant M. Group-Based Diet and Physical Activity Weight-Loss Interventions: A Systematic Review and Meta-Analysis of Randomised Controlled Trials. Appl Psychol Health Well Being 2018 Mar;10(1):62-86. [doi: 10.1111/aphw.12121] [Medline: 29446541]

13. Harden S, McEwan D, Sylvester B, Kaulius M, Ruissen G, Burke S, et al. Understanding for whom, under what conditions, and how group-based physical activity interventions are successful: a realist review. BMC Public Health 2015 Sep 24;15:958 [FREE Full text] [doi: 10.1186/s12889-015-2270-8] [Medline: 26404722]

14. Ells LJ, Radley D, Matu J, Clare K, Blackshaw J, Feeley A, et al. Supporting weight management services during the COVID-19 pandemic: Phase I insights. London, UK: Public Health England; 2020 Sep. URL: https://assets. publishing.service.gov.uk/government/uploads/system/uploads/attachment data/file/915274/WMS Report.pdf [accessed 2021-03-15]

15. Sorgente A, Pietrabissa G, Manzoni G, Re F, Simpson S, Perona S, et al. Web-Based Interventions for Weight Loss or Weight Loss Maintenance in Overweight and Obese People: A Systematic Review of Systematic Reviews. J Med Internet Res 2017 Jun 26;19(6):e229 [FREE Full text] [doi: 10.2196/jmir.6972] [Medline: 28652225]

16. Sorgente A, Pietrabissa G, Manzoni GM, Re F, Simpson S, Perona S, et al. Web-Based Interventions for Weight Loss or Weight Loss Maintenance in Overweight and Obese People: A Systematic Review of Systematic Reviews. J Med Internet Res 2017 Jun 26;19(6):e229 [FREE Full text] [doi: 10.2196/jmir.6972] [Medline: 28652225]

17. Alnuaimi A, Rawaf S, Hassounah S, Chehab M. Use of mobile applications in the management of overweight and obesity in primary and secondary care. JRSM Open 2019 Mar;10(3):2054270419843826 [FREE Full text] [doi: 10.1177/2054270419843826] [Medline: 31057801]

18. Milne-Ives M, Lam C, De Cock C, Van Velthoven MH, Meinert E. Mobile Apps for Health Behavior Change in Physical Activity, Diet, Drug and Alcohol Use, and Mental Health: Systematic Review. JMIR Mhealth Uhealth 2020 Mar 18;8(3):e17046 [FREE Full text] [doi: 10.2196/17046] [Medline: 32186518]

19. Willis EA, Szabo-Reed AN, Ptomey LT, Steger FL, Honas JJ, Washburn RA, et al. Do weight management interventions delivered by online social networks effectively improve body weight, body composition, and chronic disease risk factors? A systematic review. J Telemed Telecare 2017 Feb;23(2):263-272 [FREE Full text] [doi: 10.1177/1357633X16630846] [Medline: 26880695]

20. Shamseer L, Moher D, Clarke M, Ghersi D, Liberati A, Petticrew M, PRISMA-P Group. Preferred reporting items for systematic review and meta-analysis protocols (PRISMA-P) 2015: elaboration and explanation. BMJ 2015 Jan 02;350:g7647 [FREE Full text] [doi: 10.1136/bmj.g7647] [Medline: 25555855]

21. Michie S, Richardson M, Johnston M, Abraham C, Francis J, Hardeman W, et al. The behavior change technique taxonomy (v1) of 93 hierarchically clustered techniques: building an international consensus for the reporting of behavior change interventions. Ann Behav Med 2013 Aug;46(1):81-95. [doi: 10.1007/s12160-013-9486-6] [Medline: 23512568]

22. Higgins JPT, Altman DG, Gøtzsche PC, Jüni P, Moher D, Oxman AD, Cochrane Bias Methods Group, Cochrane Statistical Methods Group. The Cochrane Collaboration's tool for assessing risk of bias in randomised trials. BMJ 2011 Oct 18;343:d5928 [FREE Full text] [doi: 10.1136/bmj.d5928] [Medline: 22008217]

23. Risk of Bias 2 (RoB 2) Tool. Cochrane Methods. URL: https://methods.cochrane.org/risk-bias-2 [accessed 2020-12-17]

24. Sterne J, Hernán MA, Reeves B, Savović J, Berkman N, Viswanathan M, et al. ROBINS-I: a tool for assessing risk of bias in non-randomised studies of interventions. BMJ 2016 Oct 12;355:i4919 [FREE Full text] [doi: 10.1136/bmj.i4919] [Medline: 27733354] 


\section{Abbreviations}

MeSH: Medical Subject Headings

PICOS: Population, Intervention, Comparator, Outcome, and Study

PRISMA-P: Preferred Reporting Items for Systematic Reviews and Meta-Analyses Protocols

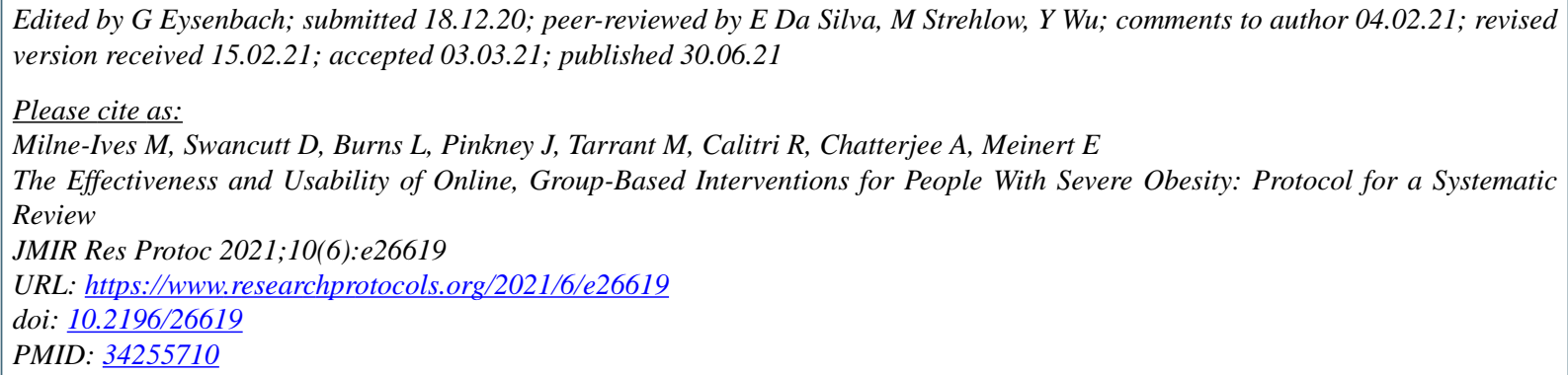

CMadison Milne-Ives, Dawn Swancutt, Lorna Burns, Jonathan Pinkney, Mark Tarrant, Raff Calitri, Arunangsu Chatterjee, Edward Meinert. Originally published in JMIR Research Protocols (https://www.researchprotocols.org), 30.06.2021. This is an open-access article distributed under the terms of the Creative Commons Attribution License (https://creativecommons.org/licenses/by/4.0/), which permits unrestricted use, distribution, and reproduction in any medium, provided the original work, first published in JMIR Research Protocols, is properly cited. The complete bibliographic information, a link to the original publication on https://www.researchprotocols.org, as well as this copyright and license information must be included. 